\title{
Bolognaprozess und Bachelor-/Masterabschlüsse: Worauf müssen sich Unternehmen einstellen?
}

\author{
Gerlach, Gerald ${ }^{1,2}$ und Schanz, Michael ${ }^{1}$ \\ ${ }^{1}$ VDE, Ausschuss Ingenieurausbildung, Stresemannallee 15, 60596 Frankfurt am Main \\ ${ }^{2}$ Technische Universität Dresden, Institut für Festkörperelektronik, 01062 Dresden
}

\section{Der Bologna-Prozess}

1999 wurde die "Bologna-Erklärung" von einem Großteil der europäischen Bildungsminister mit dem Ziel unterzeichnet, einen international wettbewerbsfähigen, europäischen Hochschulraumes bis zum Jahre 2010 zu schaffen [1]. Mit der generellen Einführung eines zweigliedrig gestuften Studiensystems sollten entsprechend gestufte Abschlüsse verständlicher und besser vergleichbar und so die Mobilität von Studierenden und Arbeitnehmern unterstützt werden. Die Berechnung des Studieraufwandes von Lehrveranstaltungen auf der Basis von sogenannten ECTS-Punkten, die Beschreibung von Studienzielen und Lehrinhalten in "Diploma Supplements" und Modulbeschreibungen sollten zu einer besseren Möglichkeit der Anerkennung von Studienleistungen an anderen in- und ausländischen Hochschulen führen. Prinzipiell wurde allerdings nicht angestrebt, eine Gleichheit der Abschlüsse der beteiligten Länder herbei zu führen.

Die Umsetzung dieser Zielstellung hat nun zu einer tiefgreifenden Umgestaltung der Studiengangslandschaft an den deutschen Hochschulen geführt. Ein großer Teil der Diplomstudiengänge in den Ingenieurwissenschaften ist bereits auf das gestufte konsekutive System umgestellt worden, wobei die beiden Stufen als Bachelor- und Master-Studium bezeichnet werden. Im Bologna-Prozess selbst wird dabei nur von „First Cycle“ und "Second Cycle" gesprochen. Die neu eingeführten, gestuften Abschlüsse tragen nicht in allen Ländern die Bezeichnungen "Bachelor" und "Master" (typischerweise mit der Ergänzung „of Engineering“ oder „of Science“). Daneben ist die Umstellung mit der Modularisierung der Lehreinheiten, mit der Vergabe von Leistungs- bzw. ECTS ${ }^{1}$-Punkten und mit einer Orientierung auf ein zu definierendes Ziel des Studiums (Outcome-Orientierung) verbunden. Zur Sicherung einer bestimmten Mindestqualität und der Studierbarkeit in der Regelstudienzeit sind diese Studiengängen regelmäßig aller fünf Jahre auf Grundlage einer „Peer Group“-basierten Begutachtung von Akkreditierungsagenturen zu akkreditieren.

\section{Hochschulausbildung in Deutschland}

Die Ingenieurausbildung in Deutschland genießt international einen sehr guten Ruf. Sie zeichnet sich durch eine klare Gliederung zwischen forschungsorientierten Studiengängen an Universitäten, anwendungsorientierten Studiengängen an den Fachhochschulen und dualen Ausbildungsgängen an Berufsakademien aus. Innerhalb der Hochschularten (Unis, FH) ist die Streuung der Qualität der deutschen Hochschulen bisher viel geringer als in vielen anderen Ländern Europas und der Welt und die Abschlüsse damit viel einfacher vergleichbar. Dabei gibt es selbstverständlich Qualitätsunterschiede zwischen den Hochschulen, wie verschiedene Rankings ausweisen [2]. Die relativ geringe Streuung in der Qualität der Hochschulen hat für die Unternehmen bei der Rekrutierung von Studienabsolventen erhebliche Vorteile, da die genaue Einordnung jedes einzelnen Studiengangs jeder einzelnen Hochschule nur bedingt notwendig war. Völlig anders ist die Situation beispielsweise in den USA, wo das Spektrum in der Qualität der Universitäten (von den Ivy League Universitäten bis hin zu den Community Colleges) deutlich breiter ist, so dass die Qualität eines Studienabschlusses nur im Zusammenhang mit der Hochschule, an der er erreicht worden ist, erfolgt (einfach sichtbar bei Doktorgraden, bei denen immer angegeben wird, von welcher Universität er verliehen wurde).

In Deutschland ist weiterhin bedeutsam, dass der Titel "Diplom-Ingenieur" bisher sowohl den akademischen Abschlussgrad als auch den Beruf bezeichnet. Dies unterstreicht das große Vertrauen in die Berufsbefähigung deutscher Ingenieure.

\footnotetext{
${ }^{1}$ ECTS - European Credit Transfer System
} 
Das Prinzip der Diplom-Ingenieurausbildung in der Elektrotechnik umfasste zunächst ein zumeist viersemestriges Grundstudium, das mit dem Vordiplom abgeschlossen wurde. Das Fachstudium, das danach in 5 bis 6 Semestern eine Spezialisierung in einer oder mehreren Fachrichtungen vorsah, sicherte sowohl eine große Breite in der Grundlagen- und Methodenausbildung als auch eine ausreichende Tiefe in der gewählten Fachrichtung. Dies sollte die Grundlage sein, mit der sich Ingenieure problemlos in angrenzende oder auch neue Fachgebiete einarbeiten können sollten.

Der VDE ist bestrebt, die Stärken des traditionellen Diplom-Systems soweit wie möglich zu erhalten und gleichzeitig diejenigen Vorteile, die ein gestuftes System bietet, zu nutzen. Eine Umstellung der Studiengänge bietet darüber hinaus die Chance - völlig unabhängig von der eigentlichen Strukturreform - qualitätssichernde Maßnahmen in stärkerem Maße einzuführen und Curricula zu überarbeiten bzw. besser aufeinander abzustimmen [8].

\section{Umstellung der Hochschulausbildung in Deutschland auf das gestufte Bachelor/Master-System}

Gegenwärtig ist zu verzeichnen, dass ein immer größer werdender Anteil der Studiengänge auf das konsekutive Bachelor/Master-Studienmodell umgestellt wurde. Eine u.a. vom VDE beauftragte Studie der Hochschul Informations System GmbH (HIS) untersuchte 2007 den aktuellen Stand des BolognaProzesses in der Elektrotechnik/Informationstechnik und dem Maschinenbau in Deutschland [3]. Von den 850 antwortenden Professoren waren 57\% eher den Reformskeptikern zuzuordnen. Trotz der anhaltenden Skepsis waren bis Ende 2007 80\% der Studiengänge in der Elektrotechnik/ Informationstechnik und dem Maschinenbau auf das gestufte System umgestellt.

Die Fachhochschulen haben die Diplomstudiengänge bei der Umstellung von meistens 8 Semestern Dauer größtenteils auf 7 Semester gekürzt. Dies entspricht der vom VDE empfohlenen Mindestdauer von berufsbefähigenden Bachelorstudiengängen. Dafür mussten Fächer (z.B. Konstruktionslehre, Technische Mechanik und Werkstoffkunde) teilweise oder ganz aus dem Curriculum genommen, Zeiten der Abschlussarbeit gekürzt oder auch Praktikumszeiten verringert werden. Zusätzlich haben die Fachhochschulen begonnen, anwendungsorientierte und teilweise auch forschungsorientierte Masterstudiengänge einzuführen, die mit einem Master of Science abschließen.

Bei den Universitäten hat sich die Gesamtstudiendauer von Bachelor/Master im Vergleich zum Diplom nicht verringert und beträgt 9 oder 10 Semester. Die Universitäten betrachten den Master-Abschluss als Regelabschluss ihrer Studiengänge und sehen den Bachelor nur als „Drehscheibe“ für den Wechsel der Studenten zwischen verschiedenen Fachrichtungen oder zwischen unterschiedlichen Hochschulen an. Bei der Umstellung auf gestufte Studiengänge entbrannte eine intensive Diskussion um das Modell "6+4" oder "7+3" (Semester in der Bachelor/Masteraufteilung). Bei den sechssemestrigen Bachelor-

Studiengängen ist häufig zu beobachten, dass das Studium stark grundlagenlastig ist und es deshalb nicht den selben Grad an Berufsbefähigung erreicht wie ein siebensemestriger Bachelor-Studiengang an Fachhochschulen. Ein sechssemestriger Bachelorabschluss wird von vielen Universitäten deshalb bevorzugt, weil dann das Masterstudium die maximale Dauer von 4 Semestern haben kann (Die Dauer konsekutiver Bachelor/Masterstudiengänge darf insgesamt 10 Semester nicht überschreiten). Die vier Semester Masterstudium werden als notwendig erachtet, wenn auch ausländische Bachelorabsolventen als Masterstudenten die Ausbildungsziele des Masterstudiums erreichen sollen.

\section{Weitere Änderungen durch das gestufte Bachelor/Master-System}

Neben der vorgeschriebenen Akkreditierung neuer gestufter Studiengänge, die ein Mindestmaß an Qualität sichern soll, wurde der Bologna-Prozess in der Regel zum Anlass genommen, um eine Reihe qualitätsverbessernder Maßnahmen in die Studiengänge einzuführen. Das betrifft insbesondere die Sicherung der Studierbarkeit der Studiengänge innerhalb der Regelstudienzeit, den Praxisbezug, den Einsatz neuer Lehr- und Lernmethoden einschließlich zusätzlicher Angebote zur Vermittlung von Zusatzkompetenzen, die Verbesserung der didaktischen Schulung von Professoren sowie den Ausbau von Beratung und Betreuung vor Studienaufnahme, während des Studieneinstiegs sowie im weiteren Studienverlauf. Besonderer Handlungsbedarf besteht nach Meinung des VDE auf dem letzteren Punkt, da die Ingenieurstudiengänge - hier auch Elektrotechnik/Informationstechnik - hohe Abbrecherquoten aufweisen [4]. Gründe hierfür liegen zwar auch in den sinkenden mathematisch-physikalischen Fähigkeiten von Studienanfängern, wie objektive Untersuchungen belegen [5] und wie es auch in den Hochschulen selbst beobachtet wird, jedoch muss dieser Entwicklung mit begleitenden Maßnahmen begegnet werden [6].

Der Bologna-Prozess bietet Chancen zur Flexibilisierung der Studienbiografie und für eine höhere Durchlässigkeit des Bildungssystems. Z.B. kann man mit einem Bachelorabschluss zunächst in den Beruf 
einsteigen und erst später ein Master-Studium anschließen, wobei dieses auch in einem angrenzenden Fach liegen kann. Eine weitere Öffnung des Bildungssystems ist zu erwarten, da durch die Anerkennung von beruflichen Leistungen die Zulassung zu Studiengängen erleichtert sowie eine Anrechnung von beruflicher Tätigkeit auf Studieninhalte möglich wird.

\section{Gleiche Abschlüsse = Alles gleich?}

Bei allen Chancen, die der Bologna-Prozess bietet, geht der VDE davon aus, dass die Reform eine schier unüberschaubare Vielfalt an Studienabschlüssen hervor bringen wird. Fachhochschulen und Universitäten bieten sowohl Bachelor- als auch Master-Studiengänge an. Die Ausrichtung von Studiengängen an Fachhochschulen ist teilweise anwendungs-, aber teilweise auch forschungsorientiert. Die Stufungsmodelle an Universitäten variieren zumeist zwischen "6+4" und "7+3". Die Regelungen für Betriebspraktika sind höchst unterschiedlich. Die flexibleren Bildungswege ermöglichen vier unterschiedliche Arten von Master-Studiengängen: konsekutive, interdisziplinäre, alleinstehend nichtkonsekutive und weiterbildende. Die unterschiedlichen Zusätze für die Bachelor- bzw. Master-Grade „of Sience" (B.Sc. bzw. M.Sc.) oder "of Engineering" (B.E. bzw. M.E.) können von Hochschulen nach eigenem Ermessen vergeben werden. Der VDE rechnet wegen dieser steigenden Diversifikation der Studiengänge und des gewachsenen Wettbewerbs zwischen den Hochschulen mit einer wachsenden Bedeutung des Hochschulnamens als Marke zusätzlich zum eigentlichen Abschlussgrad.

Darüber hinaus werden neben grundständigen Studiengängen "Elektrotechnik/Informationstechnik" immer mehr sogenannte Bindestrich-Studiengänge, wie z.B. Medieninformatik, Technische Informatik, Mechatronik und Medizintechnik, oder hochspezialisierte Studienfächer, wie z.B. Regenerative Energien, angeboten. Der VDE empfiehlt deshalb, dass sowohl kombinierte als auch frühzeitig spezialisierende Studiengänge im Ingenieurbereich die Ausnahme sein sollten und dass die Mehrzahl der angebotenen Studiengänge die Elektrotechnik und Informationstechnik in ihrer gesamten Breite berücksichtigen und dies in ihrer Bezeichnung ausdrücken muss. Ingenieurstudiengänge sollten ein breites theoretisches und methodenorientiertes Fundament besitzen, das bei der Spezialisierung in einem Teilgebiet geübt und angewendet werden soll, aber auch die schnelle selbständige Einarbeitung in andere Fachgebiete ermöglichen muss. Eine Spezialisierung oder Kombination von Studiengängen, die auf einer Einengung des breiten Grundlagenfundaments beruht, kann die langfristige Berufsfähigkeit der betreffenden Absolventen verschlechtern [7].

Auch wenn mit "Bachelor" und "Master" nunmehr Abschlussbezeichnungen vergeben werden, die im Ausland bekannter als die bisherigen Diplomgrade sind, ist die Vergleichbarkeit der Studienabschlüsse innerhalb Deutschlands im Allgemeinen viel geringer geworden. Die gestiegene Unübersichtlichkeit geht einerseits zu Lasten der Studierwilligen, für die früher vor allem nur der Unterschied zwischen Fachhochschule und Universität von besonderer Bedeutung war. Andererseits müssen nun auch die Mitarbeiter in den Personalabteilungen der Unternehmen deutlich mehr Aufwand für die Bewertung eines in Deutschland erworbenen Bachelor- oder Master-Abschlusses verwenden als es bisher bei den Diplomstudiengängen der Fall war.

\section{Profilbildung an den deutschen Hochschulen}

Da die deutsche Wirtschaft nach wie vor Elektroingenieure sowohl mit anwendungsorientiertem als auch mit forschungsorientiertem Profil braucht, spricht sich der VDE für die Beibehaltung einer klaren Profilierung in diesen zwei Richtungen aus [8]. Um die Berufsbefähigung für einen Bachelor zu erreichen, ist eine siebensemestrige Hochschulausbildung notwendig, wobei neben sechs Studiensemestern auch ein Semester für integrierte Fachpraktika und für Studien- und Abschlussarbeiten erforderlich ist [9]. Universitäten sollen die Möglichkeit haben, der forschungsorientierten Masterausbildung den höchsten Stellenwert einzuräumen.

Die neu entstehende Vielfalt der Studienabschlüsse sollte auf ein übersichtliches Maß begrenzt werden. Wie bereits oben ausgeführt wurde, sollten deshalb grundständige Studiengänge Elektrotechnik/ Informationstechnik mit späterer Vertiefung die klare Mehrheit der Studiengänge mit elektrotechnischem Hintergrund bilden.

Der VDE begrüßt die stärkere Einbindung von Schlüsselqualifikationen ins Studium. Allerdings darf diese nicht zu Lasten von Fach- und Methodenwissen gehen, da Fach- und Methodenwissen essenziell notwendige Voraussetzungen für die Ingenieurarbeit sind [10]. Der VDE empfiehlt deshalb insbesondere integrierte Ansätze für den Erwerb von Zusatzkompetenzen, z.B. innerhalb von Projektarbeiten oder von Teamarbeit. 


\section{Gute Ingenieurausbildung braucht eine verbesserte Hochschulausstattung}

Qualitätssteigerung und Studierbarkeit in den Studiengängen [11] sollen die hohen Abbrecherquoten reduzieren, die Ausbildungsqualität steigern und das Studium attraktiver zu machen. Dies kann nicht ohne eine verbesserte finanzielle und personelle Ausstattung der Hochschulen geschehen. Die Politik muss dazu die der Bedeutung der Ingenieure und der Ingenieurausbildung angemessenen Ressourcen bereit stellen [12]. Um die Abbrecherquoten weiter zu reduzieren, müssen Anstrengungen unternommen werden, den Stellenwert der Mathematik und der Technik in den Schulen wieder anzuheben.

\section{Kommt nun das Aus für „den Ingenieur"?}

Absolventen von Ingenieur-Studiengängen schlossen bisher ihr Studium mit dem akademischen Grad eines Diplomingenieurs ab, verbunden mit dem Recht, diesen akademischen Grad auch als Berufsbezeichnung führen zu dürfen. Somit ist die Berufsbezeichnung "Ingenieur" Bestandteil des entsprechenden akademischen Grades. Durch die Umstellung der Diplomstudiengänge auf das Bachelor-/Mastersystem wird nunmehr der akademische Grad des Diplomingenieurs durch die akademischen Grade Bachelor bzw. Master of Science oder Bachelor bzw. Master of Engineering abgelöst. Damit verschwindet der Begriff des Ingenieurs, was in der Wirtschaft teilweise zu erheblichen Irritationen führt, da sich die neuen akademischen Grade in keiner Weise von solchen in den Natur-, Geistes-, Sozial- und Kulturwissenschaften unterscheiden.

VDE und VDI empfehlen deshalb, dass Absolventen relevanter Studiengänge auf Zeugnissen und Urkunden neben der Verleihung des akademischen Grades Bachelor bzw. Master ausdrücklich das Recht zuerkannt bekommen, die Berufsbezeichnung "Ingenieur" zu führen. Dies kann im Einklang mit der Gesetzgebung des entsprechenden Bundeslandes gegebenenfalls mit einem Bezeichnungsvorsatz erfolgen, z.B. in folgender Form: „xyz hat den Studiengang Elektrotechnik mit dem Grad Master of Science abgeschlossen und führt die Berufsbezeichnung Diplom-Ingenieur“.

\section{Literatur}

[1] www.bmbf.de/pub/bologna_deu.pdf

[2] http://ranking.zeit.de/che9/C $\mathrm{HE}$

[3] Acht Jahre nach Bologna - Professoren ziehen Bilanz. Ergebnisse einer Befragung von Hochschullehrern des Maschinenbaus und der Elektrotechnik. HIS/VDE-Studie 2007.

[4] Empfehlung des VDE-Ausschusses „Ingenieurausbildung“ für den Umgang der Hochschulen mit sich verschlechternden Eingangsqualifikationen von Studienanfängern. VDE 2008.

[5] M. Berger, A. Schwenk: „Mathematische Grundfertigkeiten der Studienanfänger der Technischen Fachhochschule Berlin und der Schüler der Bertha-von-Suttner-OG Berlin“. Global Journal of Engineering Education 5 (2001) 3, S. 251 - 258. M. Berger, A. Schwenk: Zwischen Wunsch und Wirklichkeit: Was können unsere Studienanfänger. Die Neue Hochschule 47 (2006) 6, S. 36 ff.

[6] VDE-Empfehlung zur Verbesserung der Studienberatung und des Studieneinstiegs zur Sicherung qualifizierten Ingenieurnachwuchses. VDE 2009.

[7] VDE-Empfehlung zur Entwicklung von Ingenieurstudiengängen der Elektrotechnik und Informationstechnik. Übermäßige Spezialisierung und Diversifizierung beschneidet die Nachhaltigkeit der Ingenieurausbildung. VDE 2008.

[8] VDE-Empfehlung zur Ingenieurausbildung mit gestuften Hochschulabschlüssen. Neue Chancen nutzen - Bewährtes erhalten - Eigenverantwortung stärken. VDE 2004.

[9] Empfehlung von VDE, VDEW, BITKOM und ZVEI zu Leitlinien für Bachelor und Master. Qualitätsanforderungen an Ingenieure der Elektrotechnik und Informationstechnik mit Bachelor- und masterabschlüssen. VDE 2001.

[10] Stellungnahme des VDE Ausschusses "Beruf, Gesellschaft und Technik" zu Ingenieurkompetenzen von Berufseinsteigern. VDE 2005.

[11] VDE-Empfehlung zur Entwicklung und Sicherung einer hohen Qualität in den Studiengängen Elektrotechnik und Informationstechnik an deutschen Hochschulen. VDE 2005.

[12] Droht auch den Hochschulen ein PISA-Debakel? VDE-Empfehlung zur Ausstattung der Fachbereiche Elektrotechnik, Elektronik und Informationstechnik an Hochschulen. VDE 2002.

[13] VDE-Empfehlung für die Erhaltung der Berufsbezeichnung „Ingenieur" von Absolventen elektrotechnischer Studiengänge an deutschen Hochschulen. VDE 2008.

[14] VDI-Empfehlung zum Schutz der Berufsbezeichnung Ingenieur/Ingenieurin in ingenieurwissenschaftlichen Bachelor- und Masterstudiengängen. VDI 2009.

Alle VDE-Empfehlungen sind unter www.vde.com bzw. unter http://www.vde.com/de/Karriere/Ingenieurausbildung/Seiten/Homepage.aspx zu finden. 